\title{
Spatial attention and reaction times during smooth pursuit eye movement
}

\author{
Yasuhiro Seya $\cdot$ Shuji Mori
}

Published online: 16 December 2011

(C) Psychonomic Society, Inc. 2011

\begin{abstract}
To examine the spatial shift of attention during smooth pursuit, we measured reaction times (RTs) to a visual target that appeared during pursuit. Participants pursued a moving row of circular frames and responded to a target presented within one of the frames. The results showed large RT differences between stimulus velocities up to $5 \% \mathrm{~s}$ and $10 \%$ s or above. RTs were faster for a target appearing in the pursuit direction than for one in the opposite direction. When an auditory precue was presented, the RTs during pursuit at $10 \%$ s were faster with increases in the stimulus onset asynchrony (SOA) between the cue and the target. Furthermore, RTs were faster in the cued than in the uncued direction. These results not only support the idea that RTs during pursuit reflect the operation of attention, but also suggest that attention during pursuit can be shifted by the abrupt onset of a target stimulus and/or by prior information regarding the onset of a target stimulus.
\end{abstract}

Keywords Eye movements · Visual attention - Search ·

Reaction time methods

During smooth pursuit eye movement, spatial attention plays an important role in maintaining pursuit of a moving object by attenuating the effects of retinal image motion of

\section{Y. Seya $(\bowtie)$}

Institute of Development, Aging and Cancer, Tohoku University,

4-1 Seiryo-machi, Aoba-ku,

Sendai 980-8575, Japan

e-mail: seya-yasuhiro@fennel.rcast.u-tokyo.ac.jp

S. Mori

Intelligent Science Division, Department of Informatics, Faculty of Information Science and Electrical Engineering,

Kyushu University,

Kyushu, Japan

e-mail: mori@inf.kyushu-u.ac.jp the background (e.g., Kowler, van der Steen, Tamminga, \& Collewijn, 1984). Without attention, the image motion of the background elicits optokinetic nystagmus (OKN), which acts to shift the observer's fixation in the direction of the background motion (opposite the direction of smooth pursuit), and pursuit performance deteriorates (Kerzel, Souto, \& Ziegler, 2008). To examine the effects of attention on smooth pursuit directly, many studies have measured the performance of smooth pursuit by manipulating the attentional resources available for smooth pursuit and for secondary tasks (Chen, Holzman, \& Nakayama, 2002; Hutton \& Tegally, 2005) or by shifting the spatial location of attention (Kerzel et al., 2008; Khurana \& Kowler, 1987; Madelain, Krauzlis, \& Wallman, 2005). For example, Kerzel et al. measured pursuit gains during a dual task in which participants pursued a horizontally moving stimulus while discriminating target stimuli presented above or below the pursuit stimulus. To shift the participants' attention away from the pursuit stimulus, a cue concerning the target location was presented before the onset of the target. Kerzel et al. found that the pursuit gains during the dual task decreased after the onset of the cue or the onset of the target, suggesting that a spatial shift of attention from the pursuit stimulus impaired pursuit performance.

Although the results of many studies have indicated that spatial attention contributes to maintaining the smooth pursuit of a moving object, it is not fully understood how attention is spatially distributed during smooth pursuit. Studies using a reaction time (RT) task in which a target was presented along the pursuit trajectory have suggested that, during smooth pursuit, attention moves ahead of the pursued object along the trajectory (Tanaka, Yoshida, \& Fukushima, 1998; van Donkelaar, 1999; van Donkelaar \& Drew, 2002). For instance, van Donkelaar and Drew had participants view a display containing a central cross (" $\times$ ") 
and eight peripheral circles, four placed horizontally on the right side of the cross, and four on the left. The centerto-center separation of the stimuli was $1^{\circ}$ horizontally. The stimuli moved rightward horizontally at $3^{\circ}, 5^{\circ}, 10^{\circ}$, or $15^{\circ} / \mathrm{s}$, and after a randomized period, one of the stimuli (the target) changed its shape (from an " $\times$ " to an "o," or vice versa). The participants' task was to pursue the cross accurately and to press a button when the target shape changed. The results showed that the RTs were faster for a target appearing on the right rather than the left side of the cross, particularly at high velocities (i.e., $10 \%$ and $15 \%$ ), suggesting that attention was located ahead of the pursuit stimulus along an expected pursuit trajectory. In addition, van Donkelaar and Drew found that RTs increased with increasing stimulus velocity. They explained this result on the basis of the attentional resources available for the performance of smooth pursuit (for further details, see the General Discussion).

Recently, Lovejoy, Fowler, and Krauzlis (2009) reported data indicating that attention during smooth pursuit is centered on the pursuit stimulus. They argued that previous findings of faster RTs for a target ahead of the pursuit stimulus than for a target behind it (e.g., van Donkelaar \& Drew, 2002) reflected a spatial shift of attention triggered by the abrupt onset of a target (Theeuwes, Kramer, Hahn, \& Irwin, 1998; Yantis \& Jonides, 1990) rather than the steadystate allocation of attention during pursuit. To investigate the spatial allocation of attention during pursuit, Lovejoy et al. used a non-RT task that minimizes abrupt onsets (Deubel $\&$ Schneider, 1996). They presented a horizontally arranged row of 15 figure-8 characters with center-to-center separations of about $0.6^{\circ}$ that moved horizontally at $8^{\circ}, 12^{\circ}$, or $16^{\circ} \%$ s. After a randomized period, all but one of the characters (the target) briefly changed to either a " 2 " or a " 5 ," and the target changed to either an "E" or a "3." The participants" task was to accurately pursue the central character and to report whether the target was an "E" or a "3." Lovejoy et al. found that the proportions correct peaked at the pursuit character and fell off rapidly on either side within $1^{\circ}$ or $2^{\circ}$ from the pursuit character. No apparent change in performance was observed with the different pursuit directions or velocities (cf. van Donkelaar \& Drew, 2002). They also found small but significant effects of spatial cues on the allocation of attention. They adopted a cost-benefit analysis (e.g., Posner, 1980) with a cue presented prior to the stimulus motion. The participants' performance was significantly better on trials with a valid rather than an invalid cue, but only when the valid cue was presented on the same side as the pursuit direction - that is, when the cue was presented on the right of a pursuit stimulus that moved to the right.

The findings of the aforementioned studies indicated that it is necessary to distinguish between allocation and shifts of attention when we deal with the spatial distribution of attention during smooth pursuit. In steady-state allocation, attention will be located at or in the close vicinity of a pursuit object, with a slight change in location toward an expected region of a target to be responded to (Lovejoy et al., 2009). Abrupt presentation of a new object during pursuit will initiate a shift of attention toward that object (van Donkelaar \& Drew, 2002), with the shift occurring more quickly in the direction of pursuit than in the opposite direction. Attention will also shift away from the pursuit object when prior information is given to the observer concerning the likely position of a target (Kerzel et al., 2008).

Keeping the above distinction in mind, in this study we examined the spatial shift of attention during pursuit using an RT task similar to that used in van Donkelaar and Drew (2002). We decided to do so for two reasons. First, we wanted to confirm the finding of directional asymmetry in the shift of attention - particularly, its dependence on pursuit velocity. Van Donkelaar and Drew showed that the asymmetry in shifts of attention, indicated by the RT distribution along the pursuit trajectory, changed with pursuit velocity; the fastest RTs were observed at $1^{\circ}$ to $2^{\circ}$ ahead of a pursuit stimulus moving at a velocity of $10^{\circ} / \mathrm{s}$, and at $15 \%$ the RTs became fastest at $2^{\circ}$ or $3^{\circ}$ ahead of the pursuit stimulus. With increasing stimulus velocity, attention during pursuit moves farther ahead of the pursuit stimulus. Therefore, it can be expected that asymmetry in the spatial shift of attention, if present, will become more marked with faster stimulus velocities.

Second, we wanted to test whether attention would be shifted voluntarily along the pursuit trajectory. Van Donkelaar and Drew (2002) reasoned that asymmetry in the shift of attention during pursuit was caused by the observer's expectation of the likely position of the pursuit stimulus. This is in line with a large body of evidence in which the expectation of a target position has caused a voluntary shift of attention (e.g., Doricchi, Macci, Silvetti, \& Macaluso, 2010; Downing, 1988; Lambert \& Corban, 1992; Posner, 1980). However, as noted by Lovejoy et al. (2009), the asymmetry observed by van Donkelaar and Drew was likely to be due to an involuntary shift of attention caused by the abrupt onset of the target. Kerzel et al. (2008) reported that attention shifted voluntarily toward a location indicated by a central cue, although the direction of the attentional shift was orthogonal to that of the pursuit. To obtain a better understanding of the attentional shift during pursuit, it would also be necessary to see whether a voluntary shift of attention occurred along the pursuit trajectory, independently of the involuntary shift of attention.

To address those issues, we conducted two experiments. In Experiment 1, we measured RTs with a wide range of stimulus velocities $\left(1^{\circ}-40^{\circ} / \mathrm{s}\right)$ (cf. van Donkelaar \& Drew, 2002). The range of target positions was also extended $0^{\circ}$ to $15^{\circ}$ on both the right and left sides of the pursuit stimulus, to cover a potentially wide range of asymmetries that might be 
observed at high stimulus velocities. Although RTs to a target stimulus at a large eccentricity might be limited by decreases in visual sensitivity, the effects of an attentional shift could be examined by comparing RTs between the right and left sides of the pursuit stimulus at equal eccentricities (see the Method section). In Experiment 2, we used a similar stimulus configuration and presented an auditory precue indicating whether the target stimulus would be likely to appear on the right or the left side of the pursuit stimulus. The stimulus onset asynchronies (SOAs) between the onsets of the cue and target were 50,100 , or $150 \mathrm{~ms}$. These SOAs were designed to prevent a saccade in the cued direction before the target presentation.

In both experiments, we thoroughly analyzed the observers' pursuit eye movements. Specifically, we calculated the velocity error and the position error before and after the onset of the target stimulus in order to check whether the observer was able to maintain smooth pursuit during the RT task. Kerzel et al. (2008) reported that pursuit performance was disturbed by the shift of attention to the target stimulus.

\section{Experiment 1}

\section{Method}

Participants There were 5 participants, 4 males and 1 female, with a mean age of 24.8 years and a range of 23-26 years. One of them was the first author, and the others were naive participants. They gave informed consent for this experiment before participation. All of the participants had normal or corrected-to-normal vision.

Apparatus and stimuli All stimuli were presented on a 17in. color CRT monitor (TOTOKU CV722X) with a refresh rate of $100 \mathrm{~Hz}$ controlled by a personal computer (Dell OptiPlex GX260) with a color graphic system (Cambridge Research Systems VSG2/5), which also controlled the experimental timing, RT measurement, and recording from a response box. The stimuli were viewed monocularly using the right eye from a distance of $27 \mathrm{~cm}$. Figure 1a illustrates the stimulus display of this experiment. In the display, seven red circular frames (luminance $1.7 \mathrm{~cd} / \mathrm{m}^{2}$ ) were presented on a black background (luminance below $0.13 \mathrm{~cd} / \mathrm{m}^{2}$ ). Each frame subtended $0.8^{\circ}$ in diameter. The seven frames were aligned horizontally, with a center-to-center separation of $5^{\circ}$ between neighboring ones. The frames moved back and forth at a distance of $20^{\circ}$ and a velocity of $1^{\circ}, 3^{\circ}, 5^{\circ}, 10^{\circ}$, $15^{\circ}, 20^{\circ}, 30^{\circ}$, or $40^{\circ} / \mathrm{s}$, with an additional condition in which the frames remained stationary at the center of the display. The target stimulus was a white filled circle (luminance 7.5 $\mathrm{cd} / \mathrm{m}^{2}$ ) subtending $0.4^{\circ}$ in diameter. The target stimulus moved with the same velocity and direction as the frame

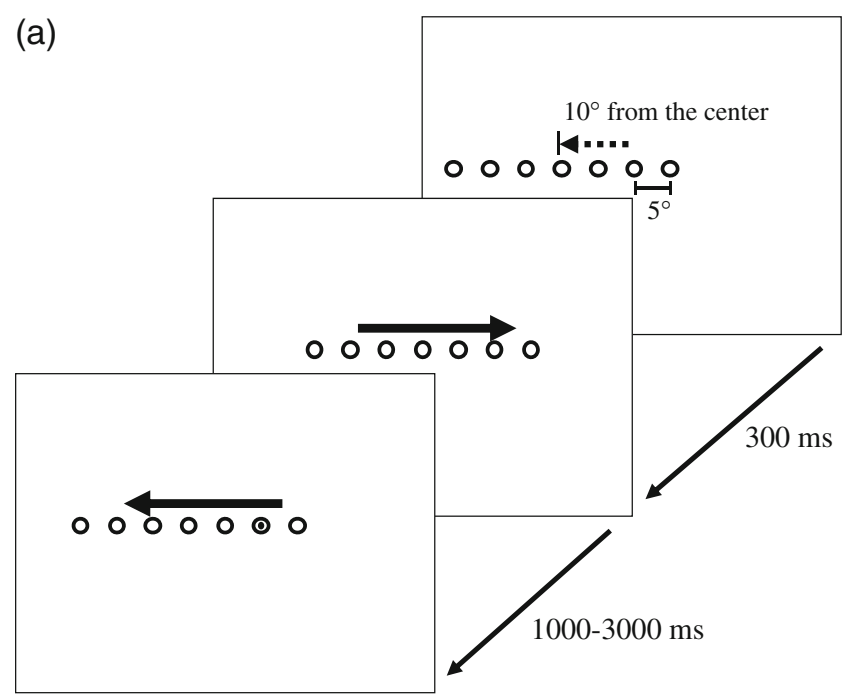

(b)

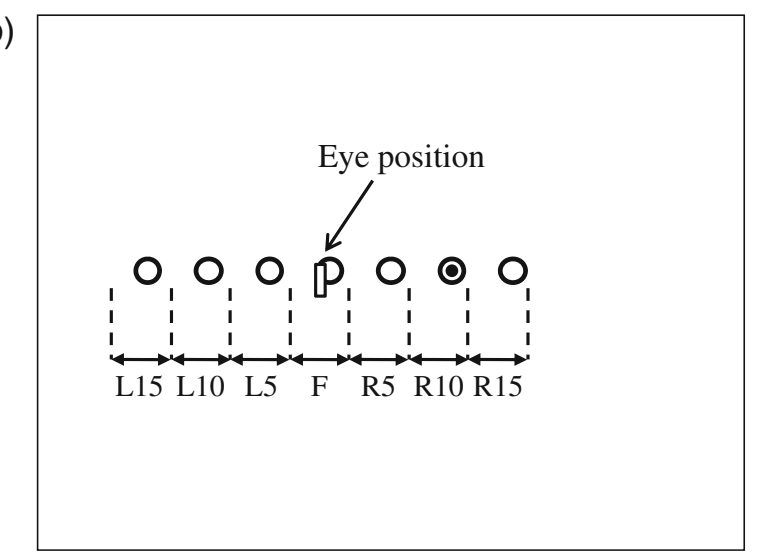

Fig. 1 Illustration of (a) the task used in Experiment 1 and (b) the range of each eccentricity. L, F, and R in the lower panel indicate left, fovea, and right, respectively

motion (it remained stationary in the stationary frame condition).

During the experiment, the eye positions were monitored by a head-mounted, video-based eyetracking system (NAC EMR-8B) that detected the foveal position from the pupil/ corneal reflection. The system had a temporal resolution of $16.67 \mathrm{~ms}$ (sampling rate of $60 \mathrm{~Hz}$ ) and could measure horizontal eye movements linearly within the monitor of the system (a range of $32^{\circ} \times 24^{\circ}$ ) with a spatial resolution of $0.1^{\circ}$ of visual angle.

Procedure The experiment was conducted in a room that was dark except for the light of the stimulus presentation. Participants were seated with their heads fixed by a chinrest and a biteboard while viewing the display. After dark adaptation for $5 \mathrm{~min}$, each experimental session was conducted. In all but the stationary frame condition, each trial began with the presentation of seven circular frames that were positioned $10^{\circ}$ to either the right or the left of the center of 
the display (see Fig. 1a). When $300 \mathrm{~ms}$ had elapsed from the onset of the frames, they started to move, followed by the target presentation at the center of one of the seven frames while the frames were moving. The foreperiod between the onset of the frame motion and the target presentation was chosen randomly for each trial from a range of 1,000-3,000 $\mathrm{ms}$. The participants pursued the central frame of the seven frames accurately and responded to the target as soon as possible by pressing the key on the response box using the index finger of their right hand. The stimulus presentation was terminated by the participant's response and was replaced with a blank display. The intertrial interval was 2,000 ms.

The moving conditions consisted of five sessions of 140 trials (two blocks of 70 trials each) for each velocity. In one block, the frames always started to move from the right of the center of the display, while in the other block they moved from the left. The order of the two blocks was randomized across sessions. Before each block, the participants were told the initial position of the central frame to be pursued. The stimulus velocity was the same throughout the five sessions. The target position was randomized across trials, with equal numbers of occurrences in all of the frames.

For the stationary frame condition, each trial began with the presentation of seven circular frames at the center of the display, followed by a randomly varied foreperiod within the range of 1,000-3,000 ms, after which the target was presented. The participants' task was identical to that in the moving frame conditions, except that the participants fixated the central frame before the onset of the target. As with the moving conditions, the stationary condition consisted of five sessions of 140 trials (two blocks of 70 trials each).

The order of the nine conditions (eight velocities for moving frames plus a stationary frame condition) was randomized across the participants. Before each velocity condition, participants were given one practice block of 70 trials. All participants completed all of the conditions over 3 or 5 days, depending on their schedule and the time available each day. They were given 2-min rest periods between sessions and 10 min between velocity conditions.

Eye movement recordings The horizontal eye positions during the stimulus presentation were recorded from participants' right eyes. The recording system was calibrated as follows. First, before each velocity condition, the participants were asked to fixate nine points on the display. Second, to check the accuracy of the recordings, before and after the experimental block, we asked the participants to fixate three points located horizontally at $0^{\circ}$ and $\pm 10^{\circ}$ from the center of the display. Fixation data to the three points were used to determine eye position on the display in the following session. All eye position data were stored on a digital tape and analyzed offline after the experiment using a computer program that calculated the retinal eccentricity of the target at the target onset and the position error and velocity error relative to the target stimulus onset.

Data analysis We excluded from subsequent analyses both the RT and eye position data obtained in trials in which the RTs were below $100 \mathrm{~ms}$ or above $1,000 \mathrm{~ms}$, and/or in which saccades were made during an interval of $100 \mathrm{~ms}$ before the target stimulus onset. To identify saccades, we used a set of velocity and acceleration criteria (e.g., Krauzlis \& Miles, 1996): A saccade was defined as an eye movement with a velocity higher than $60^{\circ} / \mathrm{s}$ (i.e., unlikely to be elicited by the pursuit stimulus) and/or acceleration higher than $2,000^{\circ} / \mathrm{s}^{2}$. The remaining data, which were slightly more than $80 \%$ of the original data across participants, were used in the following analysis.

For each participant and velocity condition, the RT data were analyzed in terms of the retinal eccentricity of the target stimulus. The retinal eccentricity of the target stimulus was first calculated by subtracting the eye position from the target position (on the display) at its onset, and the resulting eccentricity was classified into one of seven positions. Figure $1 \mathrm{~b}$ illustrates an example of the seven positions. Each position was centered at the fovea $\left(0^{\circ}\right)$ or $5^{\circ}, 10^{\circ}$, or $15^{\circ}$ to both the right and left of the fovea, with a range of $5^{\circ}$, from $-2.5^{\circ}$ to $+2.5^{\circ}$ from the central position. RTs were sorted by the corresponding eccentricity position and averaged. To examine whether spatial attention would be shifted in the direction of pursuit (Tanaka et al., 1998; van Donkelaar, 1999; van Donkelaar \& Drew, 2002), we also analyzed the RT data separately for each pursuit direction.

The eye position data of each participant were used to compute position and velocity errors. In the computation, we first removed the data corresponding to saccades and/or eye movements in the opposite direction from the pursuit stimulus motion. No interpolation was performed for the data removed by the process above. We then extracted the data contained in an 800-ms interval, from $400 \mathrm{~ms}$ before the target onset to $400 \mathrm{~ms}$ after the onset, and divided the extracted data into four 200-ms bins. Since the sampling rate of the eyetracking system was $60 \mathrm{~Hz}$ (see the Apparatus and Stimuli section above), each bin was assumed to contain 12 samples if none of them was removed. The bins that contained seven or fewer samples (about $7 \%$ of the original data) were excluded from the subsequent analyses of velocity and position errors. It should be noted that, since the pursuit stimulus (and target stimulus) disappeared after the participant's response (see the Procedure section), the eye position data beyond $400 \mathrm{~ms}$ after the onset might simply reflect perturbation of eye movements following pursuit termination. Therefore, we did not analyze the data more than $400 \mathrm{~ms}$ after the onset. 
We conducted two types of analyses on the position and velocity errors. One analysis was to calculate the position and velocity errors in each bin (e.g., Kerzel et al., 2008). The position error was computed for each sample by taking the differences between the positions of the pursuit stimulus and the eye and was then averaged over all samples in a bin. In the computation of velocity errors, the horizontal eye velocity was obtained by differentiating the eye position data and taking the average over a $200-\mathrm{ms}$ bin, and the velocity error was computed by subtracting the resulting eye velocity from the stimulus velocity. We calculated the mean of either type of error over an 800-ms interval, after removing any bins in which the error was two standard deviations beyond the mean of that interval (less than 3\%) in the original data across participants. The removal of outliers prevented the mean value from being contaminated by aberrant data, which could have occurred due to saccades related to the changes in direction of the pursuit stimulus or to blinks.

The other analysis was to calculate the means of the position and velocity errors over two bins prior to the onset of the target stimulus as a function of the eccentricity position of the target stimulus and its velocity. Fewer than $1 \%$ of the total trials across participants contained no data in any of the four bins, and this analysis was not performed on those trials.

For our statistical methods, we used nonparametric tests because of the small number of participants. Specifically, two types of nonparametric tests were performed in sequence. First, a Friedman test was performed to examine the main effects of the experimental variables - that is, velocity and eccentricity - and their interaction (Iwahara, 1964; Wilcoxon, 1949). The means of all of the variables were obtained from all 5 participants; that is, the total of 315 means $(9$ velocities $\times 7$ eccentricities $\times 5$ participants) were entered into the test after the data were divided into subsets corresponding to the levels of the variable(s) to be tested. For a main effect of velocity on RTs, for example, the data were divided into 9 subsets of 35 means ( 7 eccentricities $\times 5$ participants), from which a $\chi^{2}$ value was computed.

Second, after a statistically significant main effect was noted by the Friedman test, a Wilcoxon matched-pair signed-rank test was performed to make multiple paired comparisons for that variable. Pairs of all mean values associated with the conditions to be compared were entered into the tests. For example, pairs of 35 means ( 7 eccentricities $\times 5$ participants) were used for the paired comparisons of two different velocities. For the multiple comparisons, the overall $\alpha$ level of .05 was preserved by a Bonferroni correction - that is, by adjusting the $\alpha$ level for each comparison to $.05 / N$, where $N$ is the number of comparisons to be performed.
Results

$R T s$ Figure 2 presents the mean RTs of the 5 participants as a function of retinal eccentricity separately for the different stimulus velocities, including the stationary frame condition (the individual data are shown in Appendix A). For all of the velocity conditions, the RTs increased with increasing retinal eccentricity. On average, the differences were large between velocities of $5^{\circ}$ and $10^{\circ} / \mathrm{s}$, whereas RTs did not change much at velocities up to $5 \%$ s or $10^{\circ} / \mathrm{s}$ and above. There were large individual differences at velocities greater than $20 \%$ s (as indicated by the error bars).

There were significant main effects of retinal eccentricity, $\chi^{2}(6)=217.13$, and stimulus velocity, $\chi^{2}(8)=134.12$, both $p \mathrm{~s}<.01$, and a significant interaction between them, $\chi^{2}(48)=65.40, p<.05$. There were significant differences between all pairs of retinal eccentricities, except for the differences between left $15^{\circ}$ (L15) and right $10^{\circ}$ (R10), $\mathrm{L} 15$ and R15, and L10 and R10. The RTs were significantly faster at velocities $5 \%$ s and below than at velocities $10 \%$ and above. The RTs were also significantly different between the velocities of $1 \%$ and $5 \%$. No other differences were significant. When Friedman tests were applied to test the main effect of eccentricity at each velocity, the effect was significant at all of the velocities, all $p \mathrm{~s}<.01$.

$R T$ difference between the right and left sides Figure 3 presents the mean RT differences between the right and left sides for rightward and leftward pursuit (the individual data are shown in Appendixes B and C). Positive values mean that the RTs were faster for the target on the right than on the left, and negative values mean that the RTs were faster for the target on the left than on the right. Overall, the RTs were faster when the target was presented on the side matching the pursuit direction (the left side in leftward pursuit and the right side in rightward pursuit). For rightward pursuit

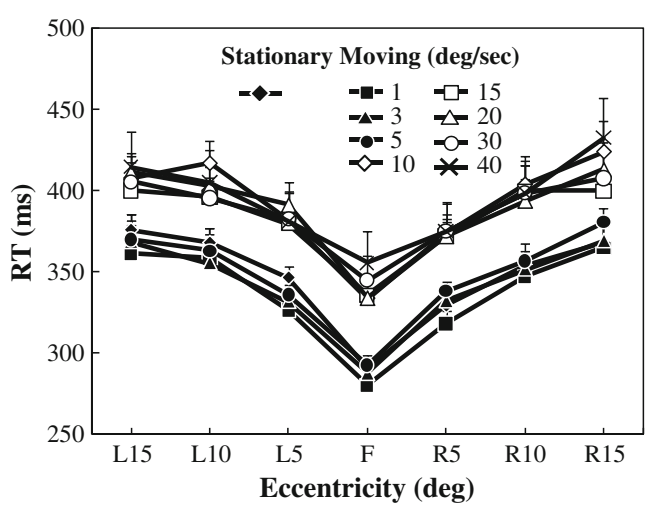

Fig. 2 Mean RTs in Experiment 1 as a function of retinal eccentricity, reported separately for different stimulus velocities, including the stationary frame condition. The vertical bars indicate the standard errors of the means 

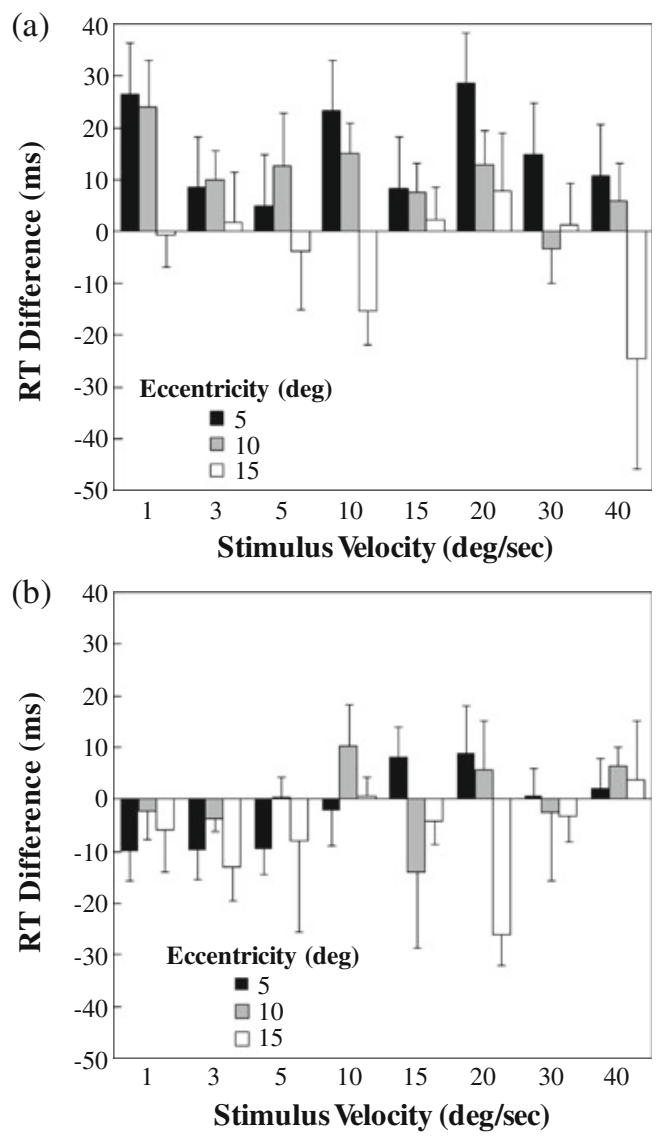

Fig. 3 Mean RT differences between the right and left sides, reported separately for (a) rightward and (b) leftward pursuit in Experiment 1. The vertical bars indicate the standard errors of the means

(Fig. 3a), the RTs were significantly faster on the right than on the left side at $5^{\circ}$ and $10^{\circ}$ of eccentricity $(p \mathrm{~s}<.05)$, but there was no difference between the two sides at $15^{\circ}$. For leftward pursuit (Fig. 3b), the difference between the two sides was significant at $15^{\circ}(p<.05)$, but not at $5^{\circ}$ and $10^{\circ}$.

Position errors Figure 4 shows single-trial recordings of horizontal eye position and the corresponding eye velocities of 1 participant during rightward pursuit at $5^{\circ}, 10^{\circ}, 20^{\circ}$, and $40 \%$ s. In any stimulus velocity condition, this participant was able to pursue the stimulus accurately and to maintain stable pursuit before the onset of the target stimulus (indicated by the arrows). Similar pursuit performance was observed in other trials used in the subsequent analyses (after the trials containing aberrant data were removed; see the Data Analysis section above) for this participant and others. Figure 5a shows the time courses of position errors of the 5 participants relative to target stimulus onset for each stimulus velocity. As can be seen in the figure, the position errors increased with increasing stimulus velocity, and at some velocities the position errors were greater at intervals after the target stimulus onset than at those before the onset. There were significant main effects of interval, $\chi^{2}(3)=$ 45.21, and stimulus velocity, $\chi^{2}(7)=125.07$, both $p$ s $<$ .01 , and a significant interaction between them, $\chi^{2}(21)=$ $37.56, p<.05$. There were significant differences between any two of the intervals, except for the differences between the intervals of -400 to $-200 \mathrm{~ms}$ and -200 to $0 \mathrm{~ms}$. There were significant differences between any two of the stimulus velocities, except for the differences between $1^{\circ}$ and $3^{\circ} / \mathrm{s}, 5^{\circ}$ and $10^{\circ} / \mathrm{s}, 5^{\circ}$ and $15^{\circ} / \mathrm{s}, 10^{\circ}$ and $15^{\circ} / \mathrm{s}, 10^{\circ}$ and $20^{\circ} / \mathrm{s}$, and $15^{\circ}$ and $20^{\circ} / \mathrm{s}$. Friedman tests applied to the data for each velocity condition showed that the position errors significantly changed with the intervals at $15 \%$ s $\left[\chi^{2}(3)=12.12\right], 20 \% \mathrm{~s}$ $\left[\chi^{2}(3)=10.68\right]$, and $40 \% \mathrm{~s}\left[\chi^{2}(3)=13.56\right.$, all $\left.p \mathrm{~s}<.05\right]$.

Figure $5 \mathrm{~b}$ shows the mean position errors of the 5 participants during the interval of $400 \mathrm{~ms}$ prior to target stimulus onset for the different retinal eccentricities and different stimulus velocities. The position errors increased with increasing stimulus velocity, whereas there was no systematic difference due to retinal eccentricity. There was a significant main effect of stimulus velocity, $\chi^{2}(7)=208.66, p<.01$, but no significant effect of retinal eccentricity, $\chi^{2}(6)=5.90$, and no significant interaction between them, $\chi^{2}(42)=44.83$. There were significant differences between any two of the stimulus velocities except for the pairs of $1^{\circ}$ and $3 \%, 5^{\circ}$ and $10^{\circ} / \mathrm{s}, 5^{\circ}$ and $15^{\circ} / \mathrm{s}, 10^{\circ}$ and $15^{\circ} / \mathrm{s}$, and $15^{\circ}$ and $20^{\circ} \%$ s.

Velocity errors Figure 6a shows the time courses of the velocity errors of all 5 participants relative to the onset of the target stimulus for each stimulus velocity (note the different scales for the panels on the left and right sides of this figure). Overall, the pattern was similar to that of the position errors. There were significant main effects of interval, $\chi^{2}(3)=49.77, p<.01$, and stimulus velocity, $\chi^{2}(7)=$ $134.60, p<.01$, and a significant interaction between them, $\chi^{2}(21)=47.88, p<.01$. The velocity errors at the last interval (200 to $400 \mathrm{~ms}$ ) were significantly higher than those at any of the earlier intervals. The velocity errors were also significantly higher at an interval of 0 to $200 \mathrm{~ms}$ than at an interval of -400 to $-200 \mathrm{~ms}$. There were significant differences between any two of the stimulus velocities, except for the differences between $1^{\circ}$ and $3 \%$. The velocity errors significantly changed with the intervals at $3 \%\left[\chi^{2}(3)=\right.$ $11.16], 5 \% \mathrm{~s}\left[\chi^{2}(3)=9.96\right], 10 \% \mathrm{~s}\left[\chi^{2}(3)=14.04\right], 15 \%$ $\left[\chi^{2}(3)=8.28\right], 20 \% \mathrm{~s}\left[\chi^{2}(3)=11.88\right]$, and $30 \% \mathrm{~s}\left[\chi^{2}(3)=\right.$ 15.00 , all $p \mathrm{~s}<.05]$.

Figure $6 \mathrm{~b}$ shows the mean velocity errors of the 5 participants during the interval of $400 \mathrm{~ms}$ before target stimulus onset for the different retinal eccentricities and different stimulus velocities. The velocity errors increased with increasing 
Fig. 4 Horizontal eye position and eye velocity of 1 participant during rightward pursuit of stimuli moving at velocities of $5^{\circ}, 10^{\circ}, 20^{\circ}$, and $40^{\circ} / \mathrm{s}$ in Experiment 1 . Gray lines indicate the actual horizontal position or velocity of the pursuit stimulus. Arrows indicate the times of target onset
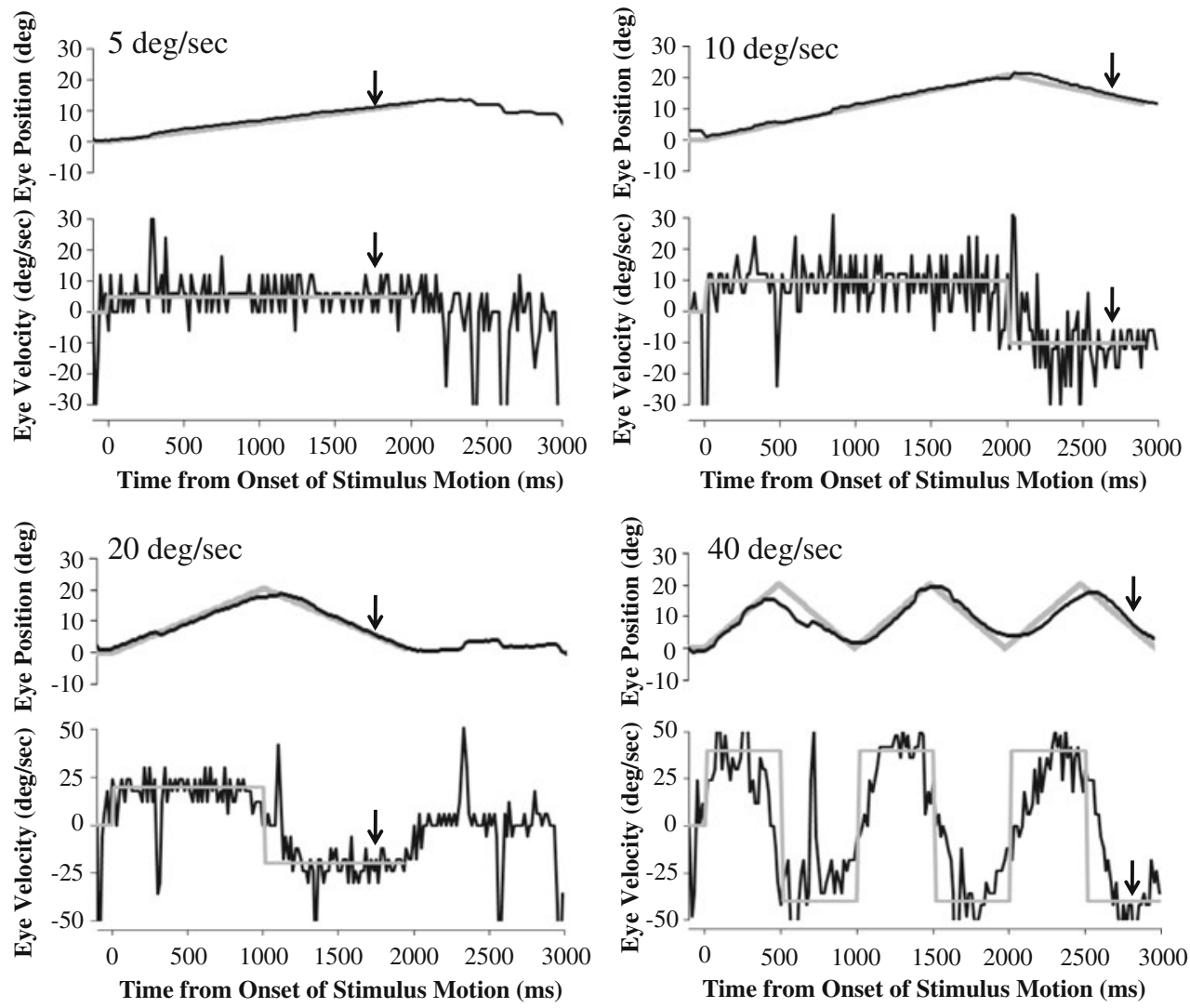

stimulus velocity, whereas there were no systematic differences due to retinal eccentricity. There was a significant main effect of stimulus velocity, $\chi^{2}(7)=233.60, p<.01$, but no significant effect of retinal eccentricity, $\chi^{2}(6)=11.08$, and no significant interaction between them, $\chi^{2}(42)=39.94$. There were significant differences between all pairs of the stimulus velocities.

(a)

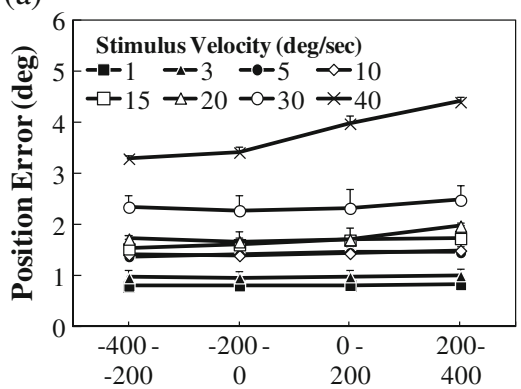

Time from onset of target stimulus (ms)

Fig. 5 Mean position errors during pursuit in Experiment 1. (a) Time courses of position errors during pursuit relative to target stimulus onset, reported separately for different stimulus velocities. (b) Mean position errors during the interval of $400 \mathrm{~ms}$ before target stimulus
Discussion

In the present experiment, the RTs increased with increasing retinal eccentricity, regardless of stimulus velocity, as in previous findings regarding RTs during fixation (Osaka, 1976, 1978; Rains, 1963). The effect of the stimulus velocity was evident in the markedly large difference between the

(b)

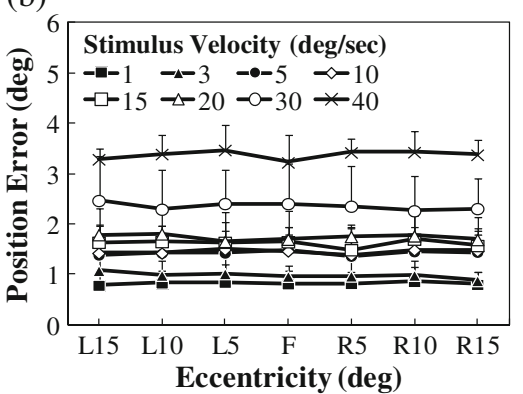

onset as a function of retinal eccentricity, reported separately for different stimulus velocities. The vertical bars indicate the standard errors of the means 
Fig. 6 Mean velocity errors during pursuit of stimuli moving at low (at or below $10 \%$, left panels) and high (at or above $15 \%$, right panels) velocities in Experiment 1. (a) Time courses of velocity errors during pursuit relative to target stimulus onset, reported separately for different stimulus velocities. (b) Mean velocity errors during the interval of $400 \mathrm{~ms}$ before target stimulus onset as a function of retinal eccentricity, reported separately for different stimulus velocities. The vertical bars indicate the standard errors of the means (a)

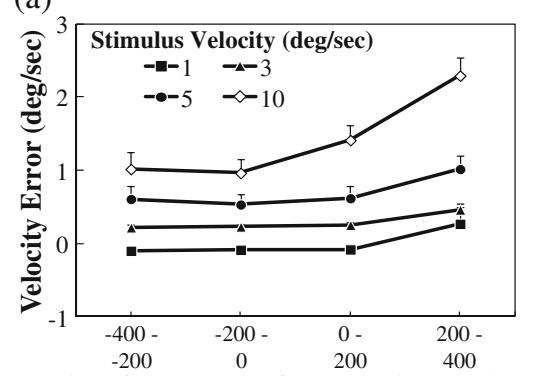

Time from onset of target stimulus (ms)

(b)

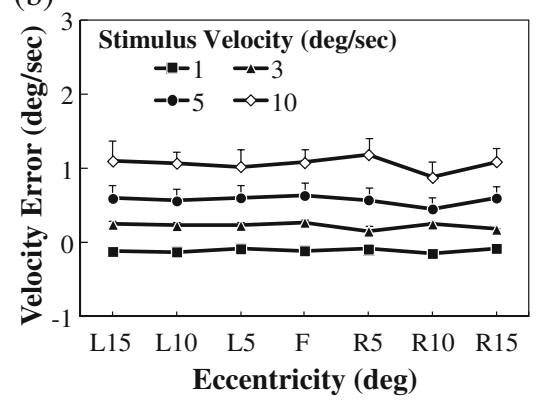

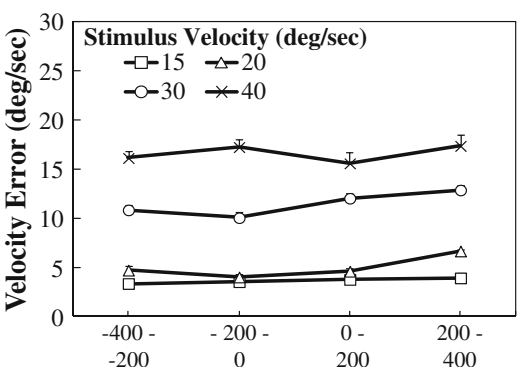

Time from onset of target stimulus (ms)

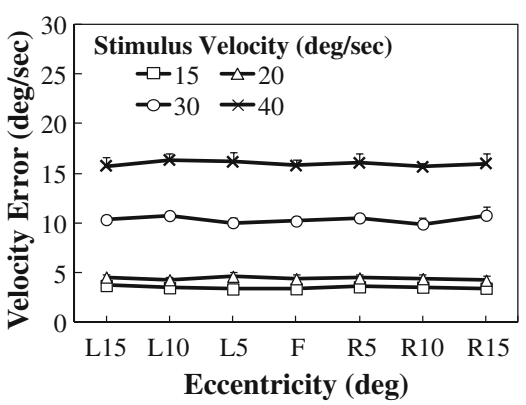

stimulus velocities up to $5 \% \mathrm{~s}$ and $10 \%$ s and above, consistent with the findings of van Donkelaar and Drew (2002). Why did the RTs rapidly increase from $5 \%$ s to $10 \%$ ? One possibility is that the participants might have switched their attentional strategy between the two velocities. At velocities up to $5 \%$ in this experiment, the participants would be able to pursue the stimulus easily and maintain a clear vision. Therefore, they might have spread their attention in order to detect the target stimulus quickly. On the other hand, at velocities of $10 \%$ s or above, the pursuing task would be more difficult than at the lower velocities, and as a result, the participants might have narrowly focused their attention on the pursuit stimulus in order to pursue the stimulus accurately. Research has shown that attentional engagement results in slower RTs for a target appearing in a new position, because extra time is required to disengage attention from the initial position before shifting to a new position (e.g., Folk \& Hoyer, 1992; Posner, 1980; Yantis \& Jonides, 1990). The size of the attentional focus may affect the time needed for the disengagement of attention from a pursuit stimulus. Several studies have reported that the effects of abrupt onsets on attentional shift become larger with an increasing size of the attentional focus (Belopolsky \& Theeuwes, 2010; Belopolsky, Zwaan, Theeuwes, \& Kramer, 2007). At velocities up to $5 \%$ in the present experiment, the participants' attention might have been disengaged rapidly by the target stimulus onset, resulting in fast RTs. On the other hand, the effect of the target stimulus onset might have been small at velocities of $10 \%$ s and above, and as a result, the participants might have needed a longer time for voluntarily disengaging their attention from the pursuit stimulus, yielding the slow RTs.

Another possibility is that, because the markedly large difference in RTs between stimulus velocities up to $5 \%$ s and $10 \%$ and above corresponded to whether or not the pursuit stimulus underwent direction reversals, the RTs might have been affected by these direction reversals. For example, the RTs might reflect the control complexity of pursuit around the direction reversals of the pursuit stimulus. At velocities of $10 \%$ s and above in this experiment, the participants had to change their pursuit direction in response to the direction reversals of the pursuit stimulus, while they did not have to do so at velocities up to $5 \%$ s. One difficulty with this explanation, however, is that van Donkelaar and Drew (2002) used a pursuit stimulus with no direction reversals and showed a large difference between $5 \%$ s and $10 \%$. These two possibilities were further examined in Experiment 2.

Spatial shift of attention in the pursuit direction The RTs during pursuit were always fastest for the target appearing at the fovea, consistent with the results of Lovejoy et al. (2009). A close comparison between the RTs for the right and left targets showed that the RTs were faster for a target in the pursuit direction than for one in the opposite direction at the equivalent retinal eccentricities of $5^{\circ}$ and $10^{\circ}$ during rightward pursuit, and at $15^{\circ}$ during leftward pursuit (Fig. 3). This result is also consistent with the findings of previous studies (Tanaka et al., 1998; van Donkelaar, 1999; 
van Donkelaar \& Drew, 2002), suggesting that participants' attention might have shifted more quickly in the pursuit direction than in the opposite direction. It is noteworthy that the RT differences between the two directions were not significant at $15^{\circ}$ during rightward pursuit and at $5^{\circ}$ and $10^{\circ}$ during leftward pursuit.

Overall, the RT advantage for a target stimulus ahead of the pursuit stimulus did not change much with increasing stimulus velocity, which is not consistent with the results of van Donkelaar and Drew (2002), who showed no RT advantages for a target ahead of the pursuit stimulus at low velocities (i.e., $3^{\circ}$ and $5^{\%} / \mathrm{s}$ ). A possible reason for the discrepancy between this finding and those of van Donkelaar and Drew was a difference in the target stimuli used. In van Donkelaar and Drew's study, the participants responded to a change in the shape of the target stimulus, while in the present experiment the participants responded to the onset of the target stimulus. Theeuwes (1990) reported that, although an abrupt change of shape captured an observer's attention, the estimated proportion of trials on which the attentional capture would occur as a result of the change was low (25\%). On the other hand, Yantis and Jonides (1984) estimated that attentional capture by an abrupt onset occurred on $90 \%$ of trials. It is possible that the target stimulus used by van Donkelaar and Drew might not have shifted the participants' attention effectively.

The results of pursuit performance showed that both position and velocity errors were significantly higher at the intervals of 0 to $200 \mathrm{~ms}$ and 200 to $400 \mathrm{~ms}$ than at the interval of -200 to $0 \mathrm{~ms}$ relative to target onset (Figs. 5a and 6a). This result is in agreement with the findings of Kerzel et al. (2008) that pursuit gains decrease when visual objects (i.e., a target stimulus or cue) appear abruptly, indicating that our participants' attention shifted away from the pursuit stimulus to the target stimulus after its onset in the present experiment. Although we did not analyze pursuit performance separately for each pursuit direction, this finding partially supports the argument of Lovejoy et al. (2009) that the RT advantage for target stimuli ahead of pursuit stimuli reported by previous studies (e.g., van Donkelaar \& Drew, 2002) was due to the spatial shift of attention rather than to the spatial allocation of attention during pursuit.

\section{Experiment 2}

Experiment 2 had two purposes. The first was to examine the effects of voluntary shifts of attention before onset of the target stimulus on RTs during pursuit (see the introduction).
If participants can shift their attention voluntarily during pursuit, RTs should be faster when the target stimulus appears in a cued direction (i.e., to the right or left of the central frame) rather than an uncued direction, independent of the target stimulus onset. The second was to examine whether RTs would become fast when participants were able to disengage their attention from a pursuit stimulus moving at $10 \%$ before onset of the target stimulus. If the markedly slow RTs at velocities of at least $10 \%$ s that we observed in Experiment 1 (Fig. 2) reflected the additional time needed for disengaging attention from the pursuit stimulus (as we discussed related to Exp. 1), then RTs during pursuit should decrease with increasing SOAs. For these purposes, an auditory precue was presented before the target presentation. The predictive validity of the cue (valid or invalid) and the SOA between the onsets of the cue and the target were manipulated.

\section{Method}

The method was similar to that of Experiment 1, except for the following changes. First, participants were presented with an auditory precue for $50 \mathrm{~ms}$ that indicated the direction of the target presentation. The auditory cues were three square tones with fundamental frequencies of 263, 527, and $1058 \mathrm{~Hz}$. The participants were told that the frequency of the auditory cue would correspond to the direction of the target onset; the lowfrequency tone indicated that the target would appear on the left side; the high-frequency tone, on the right side; and the mid-frequency tone, in the central frame. In the high- and low-frequency trials, the cue was correctly predictive for $75 \%$ of the trials (valid cue) and falsely predictive for the remaining $25 \%$ of trials (invalid cue). Many studies have used a cue validity of $75 \%-80 \%$ and have proven it useful for revealing voluntary shifts of attention (e.g., Posner, 1980; Yantis \& Jonides, 1990). Lovejoy et al. (2009) and Kerzel et al. (2008) used the same cue validity. In the middle-frequency trials, the cue was correctively predictive for $100 \%$ of the trials. We used this condition to see whether the pattern of RTs during pursuit would be consistent with that of Experiment 1 in terms of the effect of retinal eccentricity, even when the cue was presented before the target stimulus onset. The SOAs between the onsets of the cue and target were 50,100, and 150 ms. We used relatively short SOAs to prevent participants from making saccades in the cued direction before onset of the target stimulus (see the introduction), and several studies have reported that a symbolic cue effectively shifted observers' attention within less than 100 ms (Cheal \& Lyon, 1991; Müller \& Rabitt, 1989; see also Kerzel et al., 2008). 
Second, the target was presented inside one of five frames (central frame and peripheral frames located at $\pm 5^{\circ}$ and $\pm 10^{\circ}$ from the central frame) out of the seven frames in the stimulus display; that is, the leftmost and rightmost frames were not used for target presentation. In the valid cue condition of the high- and low-frequency trials, the target appeared inside either of the two frames $\left(5^{\circ}\right.$ and $\left.10^{\circ}\right)$ in the cued direction on equal numbers of trials. In the invalid cue condition, the target appeared in the uncued direction in either of the two possible frames on equal numbers of trials. In the middle-frequency trials, the target always appeared inside the central frame. Finally, the velocity of the stimuli was always $10^{\circ} / \mathrm{s}$, or the frames were stationary.

For the moving frame condition, each trial began with the presentation of stationary frames for $300 \mathrm{~ms}$. The frames then started to move horizontally back and forth for a distance of $20^{\circ}$ across the center of the display, followed by the presentation of the auditory cue. The foreperiod between the onset of frame motion and the target presentation was chosen randomly for each trial in the range of $1,000-3,000 \mathrm{~ms}$. The target stimulus was presented at one of the given SOAs from the onset of the auditory cue. The participants were asked to pursue the central frame accurately and to attend in the cued direction when the cue was presented. When the target was presented, participants were to press the button as soon as possible.

For the stationary frame condition, each trial began with the stationary frames presented at the center of the display, followed by the presentation of the auditory cue. The foreperiod between the onset of the stationary frames and the onset of the target randomly varied in the range of 1,000 3,000 ms. At a given SOA from the onset of the auditory cue, the target was presented. The participants' task was identical to that for the moving frame condition, except that the participants fixated on the central frame before the onset of the cue.

A total of 5 individuals participated, 3 males and 2 females, with a mean age of 25.6 years and a range of 23-28. Three of the participants (2 males and 1 female) had participated in Experiment 1. There were five sessions of 100 trials (two blocks of 50 trials each) for each SOA in each of the moving conditions and the stationary frame condition. Two of the participants performed the moving frame condition first, and the other three performed the stationary frame condition first. The order of the SOAs was randomized across participants. Before the experimental session, the participants were given six practice blocks of 50 trials. In all other aspects, the method was identical to that used in Experiment 1.

\section{Results}

$R T s$ Figure 7 presents the mean RTs of all 5 participants as a function of retinal eccentricity, separately for the different SOAs and trial types (valid and invalid) and for the different conditions (moving and stationary). For the moving frame condition (Fig. 7a), the RTs increased with increasing retinal eccentricity and decreased with increasing SOA. The RTs were faster for valid than for invalid trials when the target was presented on the right side, while there was no systematic difference between the two trial types when the target was presented on the left side. In the valid trials, there were significant main effects of retinal eccentricity, $\chi^{2}(4)=46.88$, and SOA, $\chi^{2}(2)=34.88$, both $p$ s $<.01$, but no significant interaction between them, $\chi^{2}(8)=7.04$. There were significant differences between all pairs of retinal eccentricities except for between L10 and R10 and between L5 and R10. RTs were significantly slower for the 50-ms SOA than for the other SOAs. In the invalid trials, there were significant

(a)

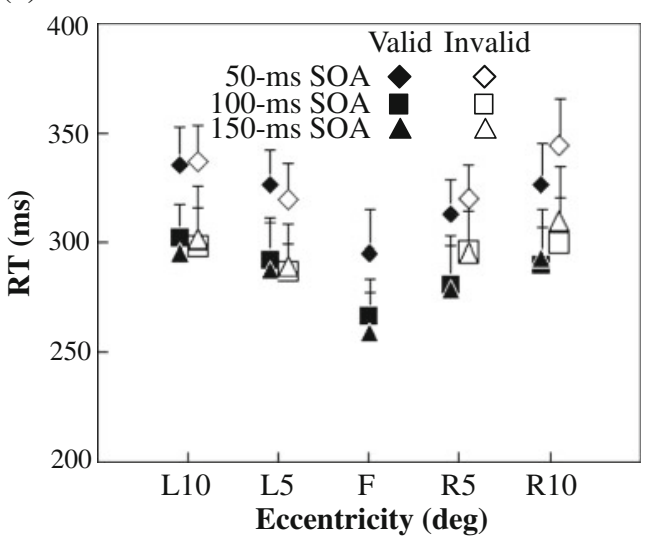

(b)

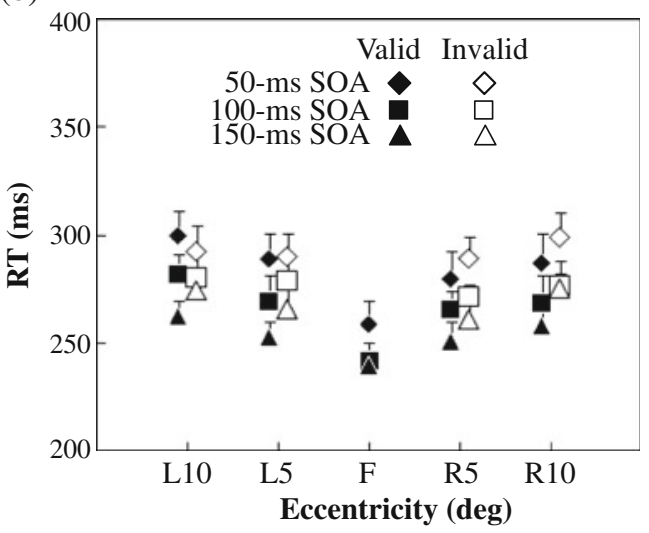

Fig. 7 Mean RTs in Experiment 2 as a function of retinal eccentricity, reported separately for different stimulus onset asynchronies (SOAs) and trial types (valid and invalid trials) in (a) the moving and (b) the stationary frame conditions. The vertical bars indicate the standard errors of the means 
main effects of retinal eccentricity, $\chi^{2}(3)=17.80$, and SOA, $\chi^{2}(2)=24.70$, both $p s<.01$, but no significant interaction between them, $\chi^{2}(6)=3.60$. The RTs were significantly slower at R10 than at either L5 or R5, and for the 50-ms SOA than for the other SOAs.

In a comparison of the valid and invalid trials, RTs were significantly faster for the valid than for the invalid trials for the 150 -ms SOA $(p<.01)$. There were no significant differences in RTs between trial types for the 50-ms and 100-ms SOAs.

For the stationary frame condition (Fig. 7b), the results were similar to those for the moving frame condition. For the RTs of valid trials, there were significant main effects of retinal eccentricity, $\chi^{2}(4)=45.60$, and SOA, $\chi^{2}(2)=21.84$, both $p \mathrm{~s}<.01$, but no significant interaction between them, $\chi^{2}(8)=9.60$. There were significant differences in all pairs of retinal eccentricities except for between R5 and R10, R5 and L5, and R5 and L10. There were significant differences between all pairs of the three SOAs. For the RTs of invalid trials, there was a significant main effect of SOA, $\chi^{2}(2)=$ $13.30, p<.01$, but no significant main effect of retinal eccentricity, $\chi^{2}(3)=6.62$, and no significant interaction between them, $\chi^{2}(6)=5.52$. RTs were significantly slower for the $50-\mathrm{ms}$ SOA than for the other SOAs.

In a comparison of the valid and invalid trials, RTs were significantly faster for valid than for invalid trials for the 100- and 150 -ms SOAs ( $p$ s $<.05$ ). There was no significant difference between the two trial types for the 50ms SOA.

Position errors Figure 8a presents the time courses of the position errors of all 5 participants relative to target stimulus onset for each SOA. As is evident in the figure, the position errors were highest at an interval of 200 to $400 \mathrm{~ms}$, and they did not differ by SOAs. There was a significant main effect of interval, $\chi^{2}(3)=37.64, p<.01$, but no significant effect of SOA, $\chi^{2}(2)=5.20$, or significant interaction between them, $\chi^{2}(6)=3.60$. There were significant differences between all pairs of intervals except between the intervals of -400 to $-200 \mathrm{~ms}$ and -200 to $0 \mathrm{~ms}$.

Figure $8 \mathrm{~b}$ shows the mean position errors during the interval of $400 \mathrm{~ms}$ before target stimulus onset as a function of retinal eccentricity, separately for the different SOAs and trial types in the moving frame condition. In the valid trials, there was no significant effect of retinal eccentricity, $\chi^{2}(4)=$ 5.39 , or SOA, $\chi^{2}(2)=5.36$, and no significant interaction between them, $\chi^{2}(8)=4.80$. In the invalid trials, there was no significant effect of retinal eccentricity, $\chi^{2}(3)=1.40$, or SOA, $\chi^{2}(2)=0.40$, and no significant interaction between them, $\chi^{2}(6)=12.48$. In a comparison of the valid and invalid trials, there was no significant difference between the trial types at any SOA.
Velocity errors Figure 8c presents the time courses of the velocity errors of all 5 participants relative to target stimulus onset for each SOA. The pattern of results was similar to that for position errors. There was a significant main effect of interval, $\chi^{2}(3)=41.48, p<.01$, but no significant effect of SOA, $\chi^{2}(2)=0.30$, or significant interaction between them, $\chi^{2}(8)=11.76$. There were significant differences between all pairs of intervals except between the intervals of -400 to $-200 \mathrm{~ms}$ and -200 to $0 \mathrm{~ms}$.

Figure $8 \mathrm{~d}$ presents the mean velocity errors during the interval of $400 \mathrm{~ms}$ before target stimulus onset as a function of retinal eccentricity, separately for the different SOAs and trial types in the moving frame condition. As can be seen in the figure, the pursuit velocity errors did not differ by condition. In the valid trials, there was no significant main effect of retinal eccentricity, $\chi^{2}(4)=8.91$, or SOA, $\chi^{2}(2)=$ 3.44 , and no significant interaction between them, $\chi^{2}(8)=$ 2.24. In the invalid trials, there was no significant main effect of retinal eccentricity, $\chi^{2}(3)=0.44$, or SOA, $\chi^{2}(2)=$ 0.10 , and no significant interaction between them, $\chi^{2}(6)=$ 5.52. In a comparison of the valid and invalid trials, there was no significant difference between the two trial types at any SOA.

\section{Discussion}

During pursuit, as well as during fixation, RTs decreased with increasing SOA (Fig. 7), which was consistent with the results of previous studies in which RTs have been measured during fixation (e.g., Folk \& Hoyer, 1992; Posner, 1980; Posner, Nissen, \& Ogden, 1978) and during pursuit (Kerzel et al., 2008). The spatial engagement of attention accounts for this result. In the present experiment, the participants focused their attention on the pursuit stimulus, whether it was moving or stationary, at the beginning of each trial. When the precue was presented, the participants had to disengage their attention and direct it to the location where the cue indicated that the target would appear. The participants then detected the target and responded to it. According to this scenario, participants' RTs obtained for short SOAs would include the time required to disengage their attention from the pursuit stimulus in addition to the time required for target detection and response execution. For long SOAs, the participants could have completed disengagement of their attention during the SOAs, resulting in faster RTs to the target presentation than for short SOAs. This is consistent with our account of the finding from Experiment 1 that markedly slow RTs at high velocities, including $10 \%$ used in this experiment, reflected the time needed for disengaging focal attention from the pursuit stimulus. It is important to note that the control complexity of pursuit (or other factors) associated with 
Fig. 8 Results for position and velocity errors during pursuit in the moving frame condition of Experiment 2. (Left) Time courses of (a) position errors and (c) velocity errors, relative to onset of the target stimulus, reported separately for different stimulus onset asynchronies (SOAs). (Right) Mean (b) position errors and (d) velocity errors during the interval of $400 \mathrm{~ms}$ before target stimulus onset, reported separately for different SOAs and trial types. The vertical bars indicate the standard errors of the means (a)

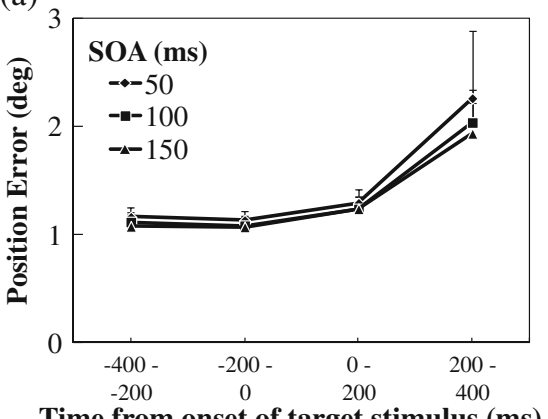

Time from onset of target stimulus (ms) (c)

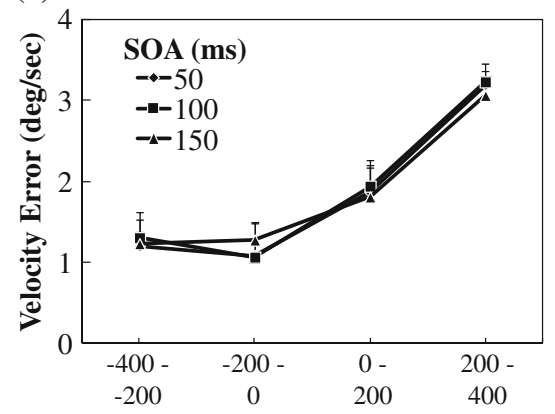

Time from onset of target stimulus (ms)

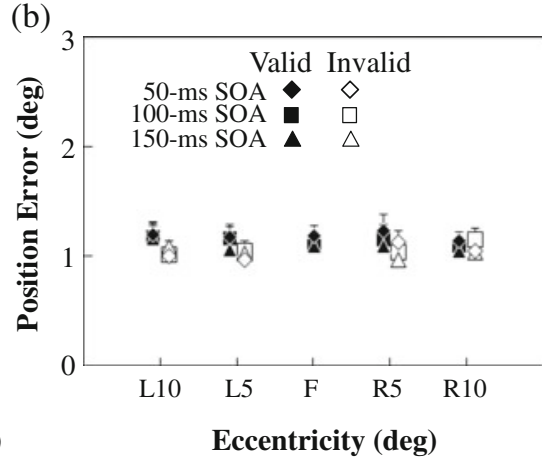

(d)

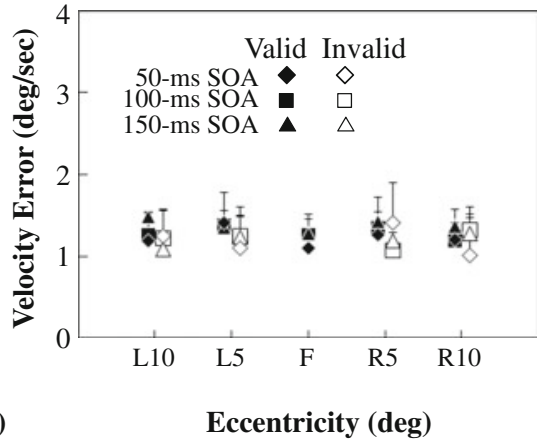

direction reversals of the pursuit stimulus would not account for the faster RTs with increasing the SOAs, because the pursuit stimulus underwent direction reversals at all of the SOAs of this experiment.

RTs were faster for the valid than for the invalid trials when the precue was presented, and the differences between the two trial types increased with increasing SOA under both pursuit and fixation conditions (Fig. 7). These results were consistent with the results of previous studies (e.g., Kerzel et al., 2008; Posner, 1980). Together with the finding that RTs decreased with increasing SOA, these results indicate that voluntary shifts of attention occur along the pursuit trajectory, independently of attentional capture by the target stimulus onset. An additional finding was that, for the moving frame condition, the RT differences between the valid and invalid conditions were smaller for the left than for the right side (Fig. 7a), although no such asymmetry between the two sides was obtained for the stationary frame condition (Fig. 7b). This finding is difficult to interpret because, to our knowledge, there is no study showing right-left asymmetry with cue validity. Further studies will be necessary to explore this asymmetry.

The results of pursuit performance showed that the position and velocity errors were significantly higher at the intervals after onset of the target stimulus ( 0 to $200 \mathrm{~ms}$ and 200 to 400 $\mathrm{ms}$ ) than at those before this onset $(-400$ to $-200 \mathrm{~ms}$ and -200 to $0 \mathrm{~ms}$ ) (Figs. 8a and c). This result is consistent with those of Experiment 1 and of recent studies (e.g., Kerzel et al., 2008) in which the spatial shift of attention away from the pursuit stimulus to the target stimulus impaired pursuit performance. The results of pursuit performance also showed that the time courses of position errors and velocity errors did not differ by SOAs, consistent with the findings of Kerzel et al., who showed that, when a symbolic cue was used, pursuit gains showed no, or only small, decreases after the cue onset.

\section{General discussion}

The present experiments clearly support the idea that RTs during pursuit are modulated by the operation of attentional shift. Experiment 1 showed distinctively slower RTs at $10 \%$ or higher velocities than at lower velocities (Fig. 2), consistent with the results of van Donkelaar and Drew (2002). Experiment 2 showed that, when a precue was presented, the RTs during pursuit at $10 \%$ secame faster as the SOAs increased (Fig. 7). Taken together, the results of the two experiments suggest that the effect of the stimulus velocity on RTs, particularly at velocities higher than or equal to $10 \%$ $\mathrm{s}$, are due to the spatial disengagement of attention from the pursuit stimulus. 
On the shift of attention during pursuit, Experiment 1 showed that RTs were faster when the target abruptly appeared in the pursuit direction, rather than in the opposite direction at equal retinal eccentricities (Fig. 3), which is consistent with the findings of previous studies (Tanaka et al., 1998; van Donkelaar, 1999; van Donkelaar \& Drew, 2002). However, the RT differences between the pursuit direction and the opposite direction did not change much with stimulus velocity (cf. van Donkelaar \& Drew, 2002). These results suggest that, while pursuing a moving object, observers shift their attention more quickly to a target stimulus onset in the pursuit direction than in the opposite direction, irrespective of stimulus velocity. Experiment 2 showed that RTs were faster for valid than for invalid trials in the pursuit condition as well as in the fixation condition (Fig. 7), suggesting that the voluntary shift of attention occurs along the pursuit trajectory. The results of Experiments 1 and 2 indicate that observers' attention during pursuit shifts away from a pursuit stimulus if there is an abrupt onset of the target stimulus and/or prior information regarding the onset of the target stimulus.

We interpreted that a switch in attentional strategy between $5 \% \mathrm{~s}$ and $10 \% \mathrm{~s}$ might have caused a delay of attentional disengagement from the pursuit stimulus, resulting in the step increase in RTs between the two velocities in Experiment 1 (see the Discussion of Exp. 1). The reason why the participants might have switched their attentional strategy between the two velocities is not clear from this study. One possibility relates to the retinal image motion during pursuit. It is well known that retinal image motion impairs visual sensitivity (Murphy, 1978; Westheimer \& McKee, 1975). Experiment 1 of the present study showed that the velocity of retinal image motion during pursuit increased with increasing stimulus velocity (see Fig. 6). The retinal image motion might have exceeded the speed at which the participants of the present study could view the pursuit stimulus without narrowly focusing their attention. As a result, the participants might have switched their attentional strategy between the stimulus velocities of $5 \% \mathrm{~s}$ and $10 \%$.

A couple of points are noteworthy concerning the methodologies used in the present study. One is that, in Experiments 1 and 2, we did not use catch trials, which are frequently used to prevent observers from anticipating the target stimulus onset (see, e.g., Buckolz \& Rodgers, 1980; Luce, 1986). However, instead of using catch trials, we randomized the foreperiod (between the stimulus motion onset and the target stimulus presentation) to minimize the participants' anticipatory responses to the target stimulus onset (e.g., Luce, 1986; Niemi \& Näätänen, 1981). Furthermore, Posner et al. (1978) used a simple RT task with no catch trials and demonstrated that the RTs clearly reflected the spatial shift of attention. Taken together, the absence of catch trials does not refute the findings on the spatial shift of attention observed in the present study, although catch trials should have been included in the task. The other point is that, because we used relatively short SOAs between the cue onset and the target stimulus onset (i.e., $50-150 \mathrm{~ms}$ ) in Experiment 2 of the present study, the auditory precue might have served as a warning signal. However, the warning effect cannot account for the results of Experiment 2 showing faster RTs in valid than in invalid trials because this effect would not differ between the two trial types. Moreover, many studies have reported that such a precue effectively shifted observers' attention voluntarily within less than $100 \mathrm{~ms}$ (e.g., Cheal \& Lyon, 1991; Müller \& Rabbitt, 1989; see also Kerzel et al., 2008). The RTs in Experiment 2 of the present study would reflect the spatial shift of attention, at least to some degree.

Although we have discussed the effect of stimulus velocity on RTs in the context of the spatial disengagement of attention, van Donkelaar and Drew (2002) argued that slower RTs with increasing stimulus velocity would be due to the attentional resources allocated for pursuit. According to their account, attentional resources would be allocated to both the pursuing and responding tasks. As the stimulus velocity increased, more resources would be needed for the pursuing task and, as a consequence, fewer resources would be allocated for the responding task, resulting in slower RTs. Several studies have supported the notion that pursuit performance and secondary (visual or auditory) tasks share a common attentional resource (Chen et al., 2002; Hutton \& Tegally, 2005; Seya \& Mori, 2007), which explains the slower RTs at $10 \%$ or higher velocities obtained in Experiment 1 of the present study. However, this explanation does not account for the result of Experiment 2 showing faster RTs with increasing SOAs, because the pursuit performance in Experiment 2 did not change much with increasing SOA.

In conclusion, the present study has demonstrated that the abrupt onset of a target stimulus and/or prior information of the onset of a target stimulus during pursuit causes a spatial shift of attention from a pursuit stimulus. This finding supports the view of Lovejoy et al. (2009) that the RT advantage for a target stimulus ahead of the pursuit stimulus reported in previous studies (e.g., van Donkelaar \& Drew, 2002) reflects the shift of attention, rather than the allocation of attention, during pursuit. In future studies, attention during pursuit needs to be examined while maintaining a clear distinction between the shift and the allocation of attention.

Author Note This research was supported by Grants-in-Aid: for Young Scientists (B) 20730485 to Y.S., for Scientific Research (B) 21330169 to S.M., and for Challenging Exploratory Research 21653078 to Kaoru Sekiyama. 


\section{Appendix A}

Fig. 9 Mean RTs for each participant in Experiment 1. The vertical bars indicate the standard errors of the means
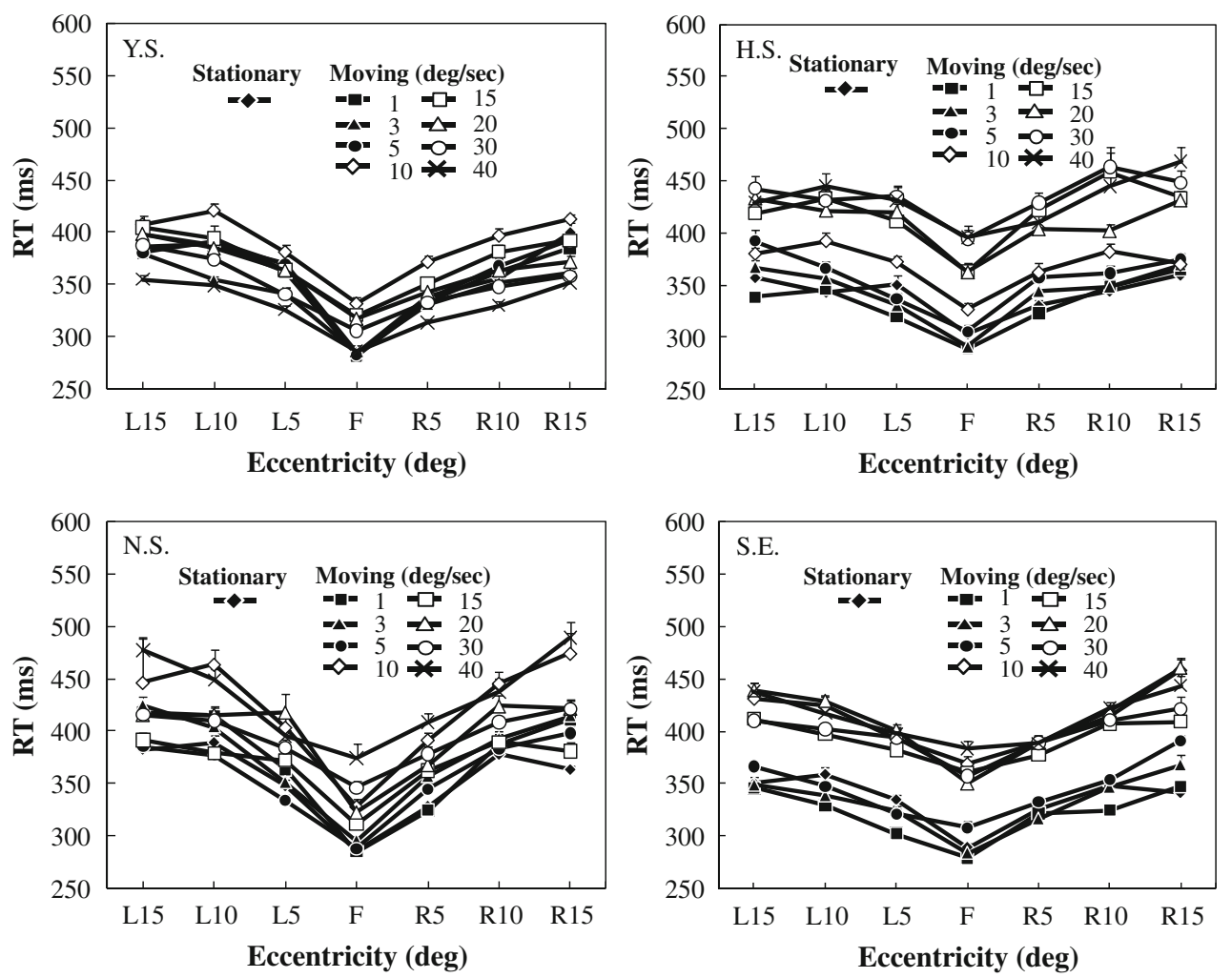


\section{Appendix B}

Fig. 10 Mean RT differences between the right and left sides during rightward pursuit for each participant in Experiment 1
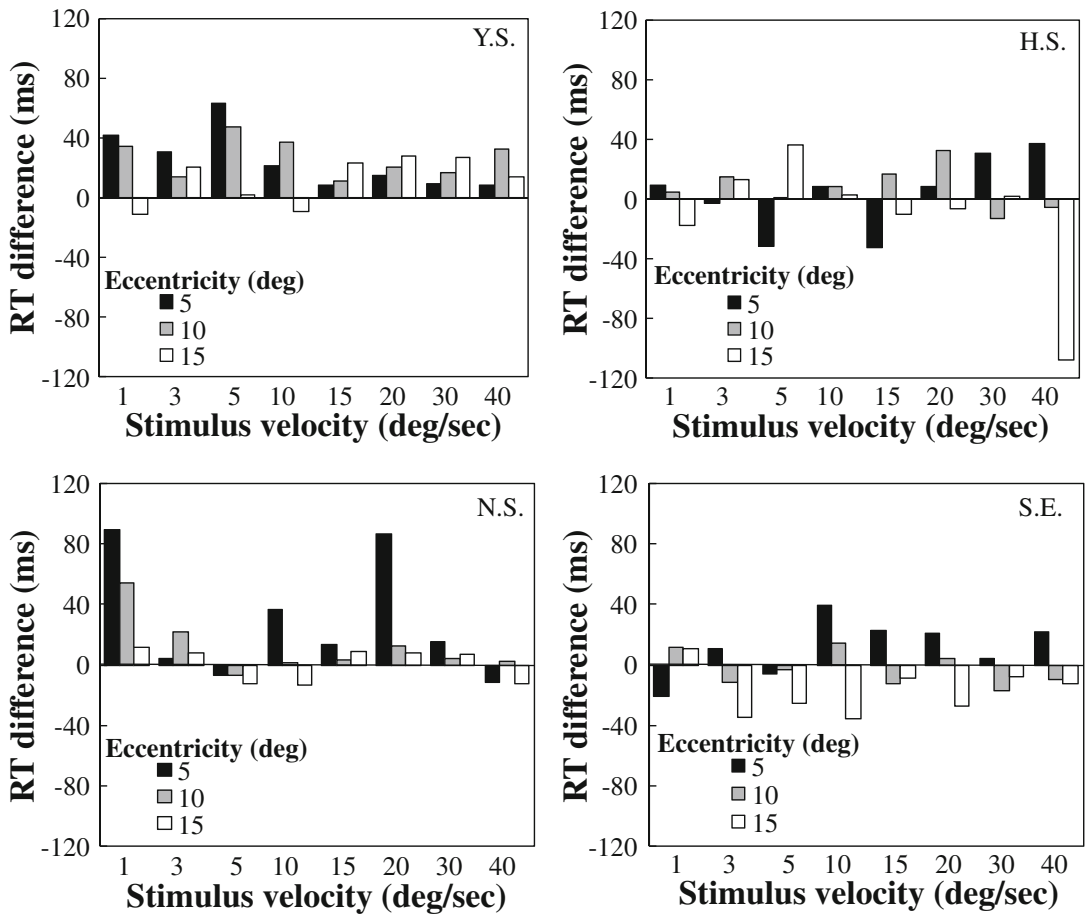


\section{Appendix C}

Fig. 11 Mean RT differences between the right and left sides during leftward pursuit for each participant in Experiment 1
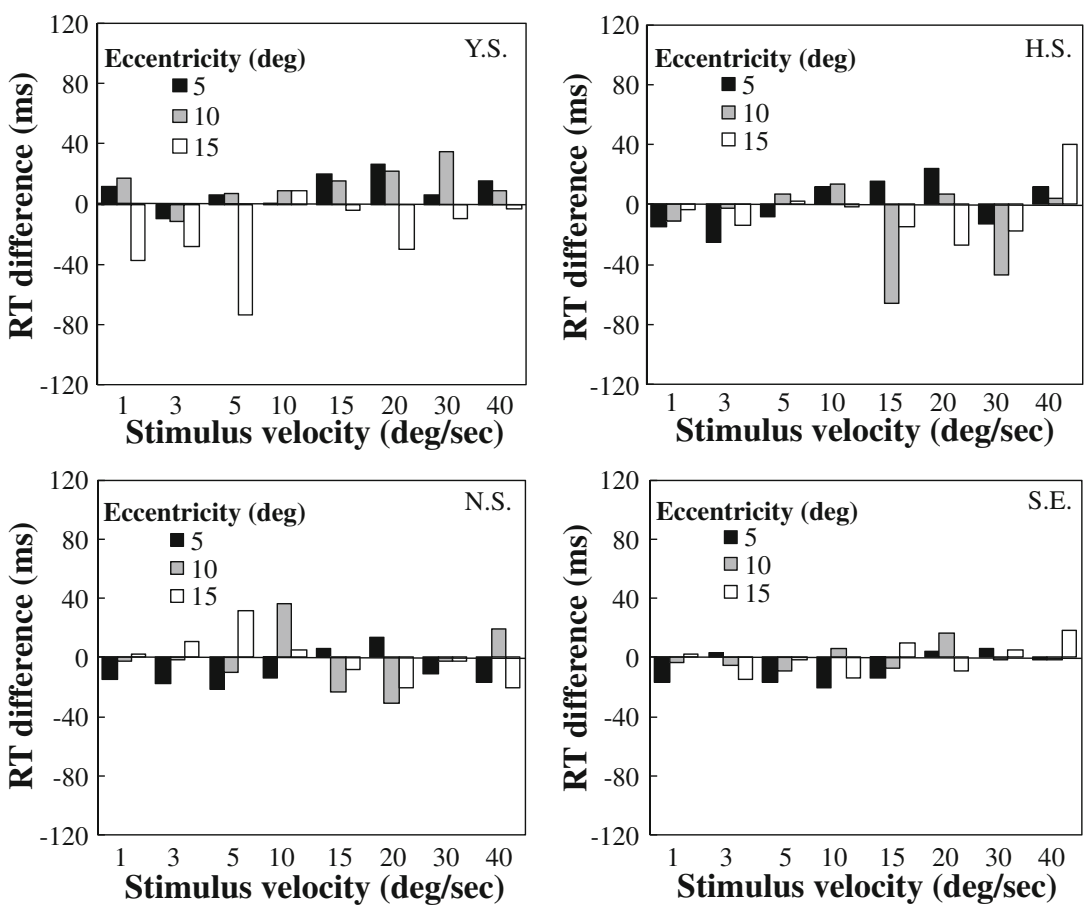

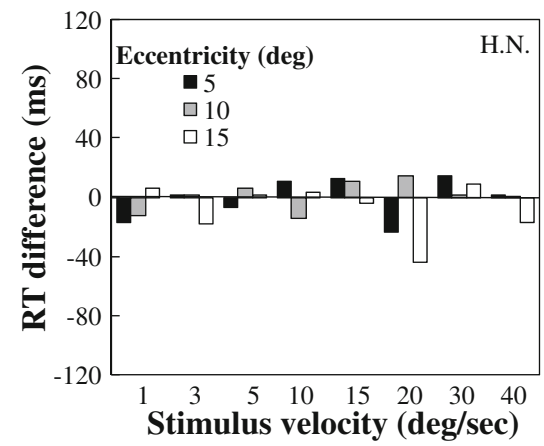

\section{References}

Belopolsky, A. V., \& Theeuwes, J. (2010). No capture outside the attentional window. Vision Research, 50, 2543-2550. doi: $10.1016 / j$.visres.2010.08.023

Belopolsky, A. V., Zwaan, L., Theeuwes, J., \& Kramer, A. F. (2007). The size of an attentional window modulates attentional capture by color singletons. Psychonomic Bulletin \& Review, 14, 934 938. doi:10.3758/BF03194124

Buckolz, E., \& Rodgers, R. (1980). The influence of catch trial frequency on simple reaction time. Acta Psychologica, 44, 191-200. doi:10.1016/0001-6918(80)90067-0

Cheal, M., \& Lyon, D. R. (1991). Central and peripheral precuing of forced-choice discrimination. Quarterly Journal of Experimental Psychology, 43A, 859-880. doi:10.1080/14640749108400960
Chen, Y., Holzman, P. S., \& Nakayama, K. (2002). Visual and cognitive control of attention in smooth pursuit. Progress in Brain Research, 140, 255-265.

Deubel, H., \& Schneider, W. X. (1996). Saccade target selection and object recognition: Evidence for a common attentional mechanism. Vision Research, 36, 1827-1837. doi:10.1016/0042-6989 (95)00294-4

Doricchi, F., Macci, E., Silvetti, M., \& Macaluso, E. (2010). Neural correlates of the spatial and expectancy components of endogenous and stimulus-driven orienting of attention in the Posner task. Cerebral Cortex, 20, 1574-1585. doi:10.1093/ cercor/bhp215

Downing, C. J. (1988). Expectancy and visual-spatial attention: Effects on perceptual quality. Journal of Experimental Psychology: Human Perception and Performance, 14, 188-202. doi:10.1037/ 0096-1523.14.2.188 
Folk, C. L., \& Hoyer, W. J. (1992). Aging and shifts of visual spatial attention. Psychology and Aging, 7, 453-465. doi:10.1037/08827974.7.3.453

Hutton, S. B., \& Tegally, D. (2005). The effects of dividing attention on smooth pursuit eye tracking. Experimental Brain Research, 163, 306-313.

Iwahara, S. (1964). Nonparametric method (in Japanese). Tokyo: Nippon Bunka Kagaku.

Kerzel, D., Souto, D., \& Ziegler, N. E. (2008). Effects of attention shifts to stationary objects during steady-state smooth pursuit eye movements. Vision Research, 48, 958-969. doi:10.1016/j. visres.2008.01.015

Khurana, B., \& Kowler, E. (1987). Shared attentional control of smooth eye movement and perception. Vision Research, 27, 1603-1618. doi:10.1016/0042-6989(87)90168-4

Kowler, E., Van der Steen, J., Tamminga, E. P., \& Collewijn, H. (1984). Voluntary selection of the target for smooth eye movement in the presence of superimposed, full-field stationary and moving stimuli. Vision Research, 24, 1789-1798. doi:10.1016/ 0042-6989(84)90010-5

Krauzlis, R. J., \& Miles, F. A. (1996). Decreases in the latency of smooth pursuit and saccadic eye movements produced by the "gap paradigm" in the monkey. Vision Research, 36, 19731985. doi:10.1016/0042-6989(95)00307-X

Lambert, A. J., \& Corban, R. (1992). Spatial attention and expectancy for colour, category and location: Further evidence against the spotlight model. Acta Psychologica, 81, 39-51. doi:10.1016/ 0001-6918(92)90010-B

Lovejoy, L. P., Fowler, G. A., \& Krauzlis, R. J. (2009). Spatial allocation of attention during smooth pursuit eye movements. Vision Research, 49, 1275-1285. doi:10.1016/j.visres.2009.01.011

Luce, R. D. (1986). Response times: Their role in inferring elementary mental organization. New York, NY: Oxford University Press.

Madelain, L., Krauzlis, R. J., \& Wallman, J. (2005). Spatial deployment of attention influences both saccadic and pursuit tracking. Vision Research, 45, 2685-2703. doi:10.1016/j.visres. 2005.05.009

Müller, H. J., \& Rabbitt, P. M. (1989). Reflexive and voluntary orienting of visual attention: Time course of activation and resistance to interruption. Journal of Experimental Psychology: Human Perception and Performance, 15, 315-330. doi:10.1037/ 0096-1523.15.2.315

Murphy, B. J. (1978). Pattern thresholds for moving and stationary gratings during smooth eye movement. Vision Research, 18, 521530. doi:10.1016/0042-6989(78)90196-7

Niemi, P., \& Näätänen, R. (1981). Foreperiod and simple reaction time. Psychological Bulletin, 89, 133-162. doi:10.1037/00332909.89.1.133
Osaka, N. (1976). Visual reaction time as a function of target size and retinal eccentricity in the peripheral visual field. Japanese Psychological Research, 18, 183-190.

Osaka, N. (1978). Naso-temporal differences in human reaction time in the peripheral visual field. Neuropsychologia, 16, 299-303. doi:10.1016/0028-3932(78)90023-4

Posner, M. I. (1980). Orienting of attention. Quarterly Journal of Experimental Psychology, 32, 3-25. doi:10.1080/0033555800 8248231

Posner, M. I., Nissen, M. J., \& Ogden, W. C. (1978). Attended and unattended processing modes: The role of set for spatial location. In H. L. Pick \& N. J. Saltzmai (Eds.), Modes of perceiving and processing information (pp. 137-157). Hillsdale, NJ: Erlbaum.

Rains, J. D. (1963). Signal luminance and position effects in human reaction time. Vision Research, 3, 239-251. doi:10.1016/00426989(63)90057-9

Seya, Y., \& Mori, S. (2007). Tradeoff between response speed and pursuit accuracy. Motor Control, 11, 109-118.

Tanaka, M., Yoshida, T., \& Fukushima, K. (1998). Latency of saccades during smooth-pursuit eye movement in man. Directional asymmetries. Experimental Brain Research, 121, 92-98.

Theeuwes, J. (1990). Perceptual selectivity is task dependent: Evidence from selective search. Acta Psychologica, 74, 81-99. doi:10.1016/ 0001-6918(90)90036-F

Theeuwes, J., Kramer, A. F., Hahn, S., \& Irwin, D. E. (1998). Our eyes do not always go where we want them to go: Capture of the eyes by new objects. Psychological Science, 9, 379-385. doi:10.1111/ 1467-9280.00071

van Donkelaar, P. (1999). Spatiotemporal modulation of attention during smooth pursuit eye movements. NeuroReport, 10, 2523 2526. doi:10.1097/00001756-199908200-00016

van Donkelaar, P., \& Drew, A. S. (2002). The allocation of attention during smooth pursuit eye movements. Progress in Brain Research, 140, 267-277.

Westheimer, G., \& McKee, S. P. (1975). Visual acuity in the presence of retinal-image motion. Journal of the Optical Society of America, 65, 847-850. doi:10.1364/JOSA.65.000847

Wilcoxon, F. (1949). Some rapid approximate statistical procedures. Stamford, CT: American Cyanamid.

Yantis, S., \& Jonides, J. (1984). Abrupt visual onsets and selective attention: Evidence from visual search. Journal of Experimental Psychology: Human Perception and Performance, 10, 601-621. doi:10.1037/0096-1523.10.5.601

Yantis, S., \& Jonides, J. (1990). Abrupt visual onsets and selective attention: Voluntary versus automatic allocation. Journal of Experimental Psychology: Human Perception and Performance, 16, 121-134. doi:10.1037/0096-1523.16.1.121 